\title{
Emperical Oral Antibiotic Therapy for Children with Low Risk Febrile Neutropenia during Cancer Chemotherapy
}

\author{
Shrestha PN ${ }^{1}$, Sah K $K^{2}$, Rana $\mathbf{R}^{3}$ \\ ${ }^{1}$ Dr. Pun Narayan Shrestha. MBBS.MD Paediatrician, Dr. Kailash Prasad Shah. MBBS.MD. Paediatric Oncologist ${ }^{2}$, \\ Dr. Rupa Rana. MBBS, Medical Officer ${ }^{3}$. Department of Oncology Kanti Children's Hospital, Kathmandu, Nepal.
}

Address for Correspondence: shrestha-pn@hotmail.com

\begin{abstract}
Introduction: In patient with fever and neutropenia during cancer chemotherapy who have a low risk of complications, oral antibiotic may be an acceptable alternative to intravenous antibiotics. Methods: We conducted a prospective hospital based study to the patients who had fever and neutropenia during caner chemotherapy. Only low risk patients i.e. neutropenia of less than seven days, ANC $>250 / \mathrm{cmm}$, without any signs of shock were included in the study. All the patients were hospitalized and given oral antibiotics Ofloxacin and Amoxy-Clav and were closely observed until fever subsided for more than 48 hours and improved from neutropenia. Results: A total of 54 cases were enrolled in the study. Out of 54 patients two patients were lost, 8 needed IV antibiotics for different reasons and 44 patients $(81 \%)$ improved well with oral antibiotics only. Conclusion: In hospitalized low risk patients who have fever and neutropenia, empirical therapy with oral ofloxacin and amoxy-clav may be a safe alternative to IV antibiotics.
\end{abstract}

Key words: Febrile Neutropenia, Cancer Chemotherapy, ANC.

\section{Introduction}

Neutropenia is a condition in which the absolute neutrohil counts (ANC) is more than two standard deviation below the normal mean. The neutrophil counts may vary on race and age. Neutropenia may be characterized as mild with an ANC of $1000-1500 / \mathrm{cmm}$, moderate $500-1000 / \mathrm{cmm}$, severe $<500 / \mathrm{cmm}^{1}$. There are various causes of neutropenia. For example:

1. Infectious causes.

a. Viral infection.

b. Bacterial infection- Typhoid fever.

c. Fungal infecton.

d. Protozoal infection.

ii. Drug Induced- Sulfa drugs, Chloramphemical, Anticonvulsant, Phenothiazines etc.

iii. Cancer Chemotherapy and Radiotherapy .

iv. Immune Mediated.

v. Hypersplenism. vi. Bone Marrow Replacement - Leukemia.

vii. Bone Marrow Failure- Hypoplastic Anemia.

viii. Neutritional Deficiency: Vit B12, Folate Deficiency.

Febrile neutropenia is one of the commonest complications of radiotherapy and chemotherapy. The immune system of children with malignancies is compromised by the anticancer drug and by the direct effect of canceritself. Inaddition, frequentIV cannulation, indwelling catheter, malnutrition, prolonged exposure to antibiotics and frequent hospitalizations all add to risk for infection in these children. Both gram positive and gram negative infections, anaerobic infection, fungal infections may occur in these patients. The lack of neutrophils can lead to a loss of inflammatory response; hence fever may be the only manifestation of infection. So in neutropenic patients, only fever warrants the use of empirical antibiotics which cover both Gram positive and Gram negative. Befor starting antibiotics we should do thorough physical examination in these patients and 
investigations like $\mathrm{CBC}$, blood culture, urine culture, $\mathrm{X}$-ray chest and swab culture from different sites of body. If culture positive and sensitivity reports are available, we have to give the antibiotic accordingly. In developed countries, about $75 \%$ of children with fever and neutropenia were ultimately found to have a documented site of infection ${ }^{2,3}$. But in our country because of different reasons isolation of organism is very limited.

In most of the centres, the patient with febrile neutropenia is treated with iv antibiotics, usually with third generation cephalosporin plus aminoglycosides. The first line antibiotic therapy should take into consideration according to the type of microbe anticipated and local resistance patterns encountered at each institution ${ }^{4}$. In addition antibiotic choice may be affected by cost, drug allergy, drug availability, renal function etc. The empirical use of oral antibiotics has been shown to be safe in low risk adults who have no evidence of bacterial focus or signs of significant illness like rigor, hypotension, and change in mental status, however substantive data for this approach is lacking in children.

There are few reports that were found regarding oral antibiotic therapy to low risk group of patient with febrile neutropenia. Alison Freifeld et.al. ${ }^{5}$ concluded that oral ciprofloxacin and amoxy-clav is safe and effective for patients with low risk febrile neutropenia. Winfried VK et al ${ }^{6}$ also reported that in low risk partients with febrile neutropenia, oral therapy with ciprofloxacin plus amoxy-clav is an effective as intravenous therapy. If we could treat patients with oral antibiotics without compromising the out come would be advantageous because oral antibiotics are cheaper, and patients would not need intravenous cannulation; hence there would be less chances for hospital acquired infections.

Aim of this research was to study the effectiveness of oral antibiotic (ofloxacin and amoxy-clav) therapy to the patient with low risk febrile neutropenia.

\section{Material and Methods}

The study was carried out in Oncology Ward of Kanti Children Hospital, from March 2007 to August 2007 i.e. duration of six months. This is a hospital based prospective study. Inclusion criteria were; children who were undergoing chemothrapy having history of documented fever with absolute neutrophil count less than $1000 / \mathrm{cmm}$ but more than $250 / \mathrm{cmm}$. Definition of fever being a peak recorded temperature of more than $38.5^{\circ}$ Celcius or two readings showing more than $38^{\circ} \mathrm{C}$ over the course of one hour. Exclusion criteria included; ANC $<250 / \mathrm{mm}^{3}$, Very sick patients - having sings of shock, altered mental function, respiratory distress, those unable to take orally and those having a history of persistent vomiting and diarrhea, and pain or abdominal tendererness. Also excluded were those who had found clear focus of infection which needed i.v. antibiotics, like meningitis and children who refused to take oral antibiotics. And finally those who were expected to have neutropenia of more than one week duration.

\section{Study Design}

A history was obtained and physical examination was performed on each patient within an hour after admission. A complete blood count, chest x-ray, routine examination of urine, blood culture, urine culture, swab cultures; if indicated, were performed before empirical therapy was begun. After the initial evaluation, patients were enrolled in the study according to above inclusion criteria (low risk group). They were treated with oral antibiotic ofloxacin and amoxy-clav. High risk patients who were not included in this study were treated with i.v. antibiotics. If $\mathrm{ANC}$ was $<250 / \mathrm{cmm}$ they were treated with i.v. antibiotic. Patients were treated until neutropenia resolved (ANC >1000) and fever subsided. The relatives or the care taker of the patients were counselled about the treatment protocol and verbal consent was also taken.

\section{Clinical Assessment}

All the patients were closely monitored by doing a thorough clinical assessment and laboratory investigations. Changes in treatment regimen into i.v. antibiotics were made if the patient did not improve (persistent high fever, persistent vomiting, ANC decreasing, features of shock present). These patients were labeled as treatment failures and i.v. antibiotics either cefotaxim and amikain or ceftazidime and vancomycin were given depending on the severity of condition.

\section{Evaluation of Response of Therapy}

Clinical outcomes were prospectively defined. Oral antibiotic therapy was considered to have been successful if the patient improved without any change of antibiotic and increased ANC. Treatment was considered to have failed if the regimen had to be modified by addition of one or more antibacterial or antifungal agents. All the patients were followed up to seven days. Statistical Analysis was done in terms of percentage effectiveness of therapy.

\section{Results}

From March 2007 to Augst 2007, a total of 54 patients were enrolled in this study. Among these patients, eight patients needed i.v. antibiotics because of the above mentioned reasons reasons. Two patients were lost to follow up. 
Table 1: Showing age distribution

\begin{tabular}{|l|l|c|}
\hline \multirow{2}{*}{ Age } & Mean & $7.2($ Years $)$ \\
\cline { 2 - 3 } & Range & $2-14$ (Years) \\
\hline
\end{tabular}

Table 2: Showing sex distribution

\begin{tabular}{|l|l|c|}
\hline \multirow{2}{*}{ Age } & Female & 32 \\
\cline { 2 - 3 } & Male & 22 \\
\hline
\end{tabular}

Table 3: Showing Characteristic of Patient

\begin{tabular}{|l|l|c|}
\hline \multicolumn{2}{|l|}{ Characteristics } & $\begin{array}{c}\text { Numbers of } \\
\text { Patients (n=54) }\end{array}$ \\
\hline \multirow{2}{*}{$\begin{array}{l}\text { Type of } \\
\text { Cancer }\end{array}$} & Haematological & 40 \\
\cline { 2 - 3 } Neutropenia & Solid Tumor & 14 \\
\cline { 2 - 3 } & ANC $>500 / \mathrm{cmm}$ & 35 \\
\hline \multirow{2}{*}{$\begin{array}{l}\text { Cause of } \\
\text { fever }\end{array}$} & Unexplained $<500 / \mathrm{cmm}$ & 17 \\
\cline { 2 - 3 } & Documented & 45 \\
\end{tabular}

Total 54 patients were enrolled in this study. Out of these, $44(81 \%)$ patients improved only with oral antibiotics and 8 patients needed i.v. antibiotics. Ampotericine B was also added in three patients. No mortality was noted in these studied patients. Two patients were lost to follow up and could not be could not be traced.

\section{Discussion}

Febrile neutropenia is usually treated with i.v. antibiotics in most of the centers. We often treat febrile neutropenia with i.v. cefotaxime plus amikacin or cefepime alone. We found that combination of oral ofloxacin and amoxy-clav were effective oral therapy to the patients with febrile neutropenia of a low risk category. Treatment was successful in nearly $80 \%$ of all episodes with oral therapy. Our definition of low risk group was taken as explained before in patients with history of fever of less than three days and those that had no features of shock, abdominal tenderness or diarrhea. The case for selection of low risk patient is extremely important. All patients with high risk febrile neutropenia must be treated with i.v. antibiotics without any delay.

In this study we gave i.v. antibiotics if $\mathrm{ANC}<250$ / $\mathrm{cmm}$. But in some studies it was found that even an ANC $<100 / \mathrm{cm}$ were successfully treated with oral antibiotics. Alison Freifeld, Donna Marchigiani et al reported that $71 \%$ of patients who were given oral antibiotic group having a mean ANC of $81 / \mathrm{cmm}$ were successfully treated with oral ciprofloxacin plus amoxy-clav ${ }^{7}$.

Similarly Winfried V. Kern, Alain Cometta and et. al. reported that $86 \%$ of their patients were successfully treated with oral antibiotics ${ }^{8}$.

Alejandria MM, Panaligan MM, Molina FG et.al., during the International Cochrane Colloquium $8^{\text {th }} 2000$, cape town, also reviewed the data regarding oral vs intravenous antibiotic for low risk febrile neutropenia and concluded that oral antibiotic regimes were equally effective as standard intravenous antibiotic therapy in the management of patient with low risk febrile neutropenia. ${ }^{\text {. }}$

Jean Klastersky, Marianne Paesmans et al studied 383 first febrile neutropenic episodes predicted at low risk of complication, 178 patients, mainly with solid tumors were treated orally; Seventy-nine patients (44\%) were discharged early (with a median time to discharge of 26 hours); no complications occurred among them except that three patients had to be readmitted, resulting in a success rate of $96 \%(95 \% \mathrm{CI}, 92 \%$ to $100 \%){ }^{10}$

Liat Vidal, Mical Paul, Itsik Ben doret al, found out that the mortality rate was similar comparing oral and IV antibiotic treatment. Treatment failure rates were also similar. No significant heterogeneity was shown for the primary outcomes. This effect was stable in a wide range of patients. Quinolones alone or combined with other antibiotics were used with comparable results. Adverse reactions, mostly gastrointestinal, were more common with oral antibiotics. ${ }^{11,12 .}$

Table 4: Outcome of Empirical Therapy with Oral Ofloxacin and Amoxy-clav.

\begin{tabular}{|l|c|c|c|c|}
\hline Characteristics. & Oral Antibiotics. & Improved. & Lost to Follow up. & $\begin{array}{c}\text { Change into IV } \\
\text { Antibiotics. }\end{array}$ \\
\hline $\begin{array}{l}\text { Unexplained Fever } \\
(\mathrm{n}=45)\end{array}$ & 45 & 40 & 2 & 3 \\
\hline Documented Fever $(\mathrm{n}=9)$ & 6 & 4 & - & 2 \\
\hline Pneumonia & 2 & - & - & 2 \\
\hline UTI & 1 & - & - & 1 \\
\hline Cellulitis & & & & \\
\hline
\end{tabular}




\section{Limitation}

This is a small study, we need to do a bigger study to verify whether or not to use oral antibiotic in febrile neutropanic patients. The study was done in only those patients who were in the low risk catagory. We had not included those patients with an ANC of less than 250/ $\mathrm{cmm}$. In some studies it was found that patients with ANC $<100 / \mathrm{cmm}$ were also treated safely with oral antibiotics.

\section{Conclusion}

Though we are treating the patient with febrile neutropenia with i.v. antibiotics as first line therapy, the larger study comparing the efficacy of the use of oral antibiotic should be done. If we could treat those patients with febrile neutropenia with oral antibiotic, it would be more cost effective and would also decrease their hospital stay. How ever we conclude that in our study we could successfully treat low risk febrile neutropenic patients with oral antibiotics.

\section{References}

1. Behrman, Kliengman and Arvin, Neutropenia, Nelson Textbook of Paediatrics $17^{\text {th }}$ Ed Saunders Elsevier p.717-20.

2. Agrawal BR, Perilongo G, Rogers P, StrahlendorfC, Eden OB. Practical Paed Oncology.International Society of Paediatric Oncology (SIOP), $2^{\text {nd }}$ Ed, 2931.

3. Behrman, Kliengman and Arvin, Febrile Neutropenia, Nelson Textbook of Paediatrics, $17^{\text {th }}$ Ed,Saunders Elsevier p.853-855.

4. Bob Phillips, Karen Selwood Sneila M Lane et al. Variation in Policies for the Management of Febrile Neutropenia in United Kingdom Children's Cancer Study Group Arch. Dis. in Children 2007,92,49598.
5. Antibacterial Prophylaxis in Patients with Cancer and Neutropenia. $N$ Eng $J$ Med 2006 Jan 5;354(1):90-4.

6. Kern WV, Cometta A, Bock Rd, Langenaeken $\mathrm{J}$ et al, Oral versus Intravenous Empirical Antimicrobial Therapy for Fever in Patients with Granulocytopenia who Are Receiving Cancer Chemotherapy. N Eng J Med 1999 Jul 5;341:312318.

7. Alison Freifeld et al double blind comparison of empirical oral and intravenous antibiotic therapy for low risk febrile patients with neutropenia during cancer therapy. $N$ Eng. $J$ of Med,1999 341,305-311.

8. Winfried V Kern, Alain et. al. Oral vs IV empirical antimicrobial therapy for fever in patient with granulocytopenia who are receiving chemotherapy. N Eng J Med, 1999, Vol.341,312-318.

9. M.M. Alejandria, M.M. Panaligan, F.G. Molina, M.F.P. Raymundo.Colloquim Abstracts. $8^{\text {th }}$ International Cochrane Colloquium. 2000, Cape Town .

10. Klastersky J, Paesmans M. et al. Outpatient Oral Antibiotics for Febrile Neutropenic Cancer Patients Using a Score Predictive for Complications. Journal of Clinical Oncology, 2006; 24:25,41294134.

11. Vidal L, Paul M,Bendor I et al. Oral versus intravenous antibiotic treatment for febrile neutropenia in cancer patients: a systematic review and meta-analysis of randomized trials. Journal of Antimicrobial Chemotherapy 2004 54(1):29-37.

12. Vidal L, Paul M, Ben-dor I. et,al. Oral vs IV Antibiotic Treatment for Febrile Neutropenia in Cancer Patients. Cochrane Database, 2004, Issue 4, Art. No: CD 003992. 